\title{
Genetic interaction between rice PLASTOCHRON genes and the gibberellin pathway in leaf development
}

\author{
Manaki Mimura and Jun-lchi Itoh
}

\begin{abstract}
Background: The rice PLASTOCHRON (PLA) genes PLA1 and PLA2 regulate leaf maturation and the temporal pattern of leaf initiation. Although the function of PLA genes in the leaf initiation process has been analyzed, little is known about how they affect leaf growth. Previously, we suggested that PLA1 and PLA2 function downstream of the gibberellin (GA) signal transduction pathway. In the present study, we examined the phenotype of a double mutant of pla and slender rice 1 (s/r1), which is a constitutive GA response mutant. By analyzing these double mutants, we discuss the relationship between PLA-related and GA-dependent pathways and the possible function of PLA genes in leaf growth.
\end{abstract}

Findings: Single s/r1 and pla mutants exhibited elongated and dwarf phenotypes in the vegetative stage, respectively. The stature and leaf size of the pla1/s/r1 and pla2/s/r1 double mutants were intermediate between those of the pla and s/r 1 single mutants. However, the effects of s/r 1 on leaf elongation were markedly suppressed in the pla1 and pla2 mutant backgrounds. On the other hand, the change in cell length in the double mutants was almost the same as that in the single mutants. An expression analysis of genes involved in GA biosynthesis and catabolism indicated that feedback regulation functioned normally in the pla/s/r1 double mutants.

Conclusions: Our genetic results confirm that PLA genes regulate leaf growth downstream of the GA pathway. Our findings also suggest that $P L A 1$ and $P L A 2$ are partly required for GA-dependent leaf elongation, mainly by affecting cellular proliferation.

Keywords: Rice; PLASTOCHRON 1; PLASTOCHRON 2; Gibberellin; SLENDER RICE 1

\section{Findings}

Rice plastochron (pla) mutants show a short plastochron and small precocious leaves. PLA1 and PLA2 encode a cytochrome P450, CYP78A11, and an RNA-binding protein, respectively. They are expressed in leaf primordia and regulate the leaf initiation rate and leaf maturation (Miyoshi et al. 2004; Kawakatsu et al. 2006). Thus, PLA1 and PLA2 play important roles in leaf development. Previously, we showed that PLA1 and PLA2 function downstream of the gibberellin (GA) signal transduction pathway (Mimura et al. 2012), and that pla1 and pla2 plants exhibited reduced sensitivity to GA treatment. In addition, GA treatment induced PLA1 and PLA2 expression. In accordance with these results, the expression

\footnotetext{
* Correspondence: ajunito@mail.ecc.u-tokyo.ac.jp

Graduate School of Agricultural and Life Sciences, University of Tokyo, Tokyo
} 113-8657, Japan

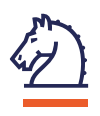

levels of PLA genes were increased in slender rice $1(\operatorname{sir} 1)$, which is a constitutively active GA signaling mutant, and decreased in $\operatorname{slr} 1-D$, which shows reduced sensitivity to GA. However, genetic evidence for the interaction between PLA genes and GA signaling genes is lacking. In the present study, we constructed pla1/slr1 and pla2/slr1 double mutants to investigate the genetic relationships between PLA genes and the GA signaling pathway.

\section{Phenotypes of pla1 and s/r1 double mutants}

slr1 is a constitutive GA response mutant that is caused by a loss-of-function of DELLA, which is a key factor in the repression of GA responses (Ikeda et al. 2001). slr1 mutants showed elongated leaves and internodes. In contrast, pla mutants showed dwarfism and small leaves. PLA1 encodes the cytochrome P450 family protein CYP78A11, which is a member of the CYP78A subfamily (Miyoshi et al. 2004). Many reports have shown that 
CYP78A family genes regulate organ growth (e.g., seed or fruit size) in several plant species (Anastasiou et al. 2007; Fang et al. 2012; Chakrabarti et al. 2013; SoteloSilveira et al. 2013). It has also been suggested that CYP78A family members are involved in producing an as yet unidentified substance that functions as a mobile growth regulator (Anastasiou et al. 2007; Adamski et al. 2009; Eriksson et al. 2010).

To determine the genetic interaction between SLR1 and $P L A 1$, we generated pla1/slr1 double mutants by crossing SLR1 heterozygous plants with PLA1 heterozygous plants. At the 3 -week-old seedling stage, the pla1/ slr1 double mutants showed intermediate phenotypes (Figure 1A-C, Table 1). However, the effects of the slr1 mutation on plant height and leaf size in the pla1 background were weaker than those in wild type. The $\operatorname{sir} 1$ plants were $53 \%$ taller than the wild-type plants, whereas the height of the pla1/slr1 double mutant was $23 \%$ that of the pla1 single mutant (Figure 1B). In terms of leaf length, the effect of the $\operatorname{slr} 1$ mutation was much more obvious in the wild-type background than in the pla1 mutant background. The third leaf sheath of $\operatorname{sir} 1$ was $123 \%$ longer than that of wild type, whereas that of the pla1/slr 1 double mutant was only $53 \%$ that of the pla 1 single mutant (Figure 1C). These results suggest that PLA1 activity is partly necessary for leaf elongation in slr1 mutant plants.

Cell size is one of the factors determining leaf size. To clarify how cell size contributes to leaf elongation in $\operatorname{slr} 1$ and pla1/slr 1 double mutants, we compared the lengths of epidermal cells on the adaxial side of the third leaf sheath in each mutant (Figure 2A). Our results indicate that the effects of the $\operatorname{sir} 1$ mutation on cell size were comparable between the wild-type and pla1 backgrounds. Cell length was increased by $17 \%$ in $\operatorname{slr} 1$ single mutant plants and by $14 \%$ in pla1/slr double mutant plants
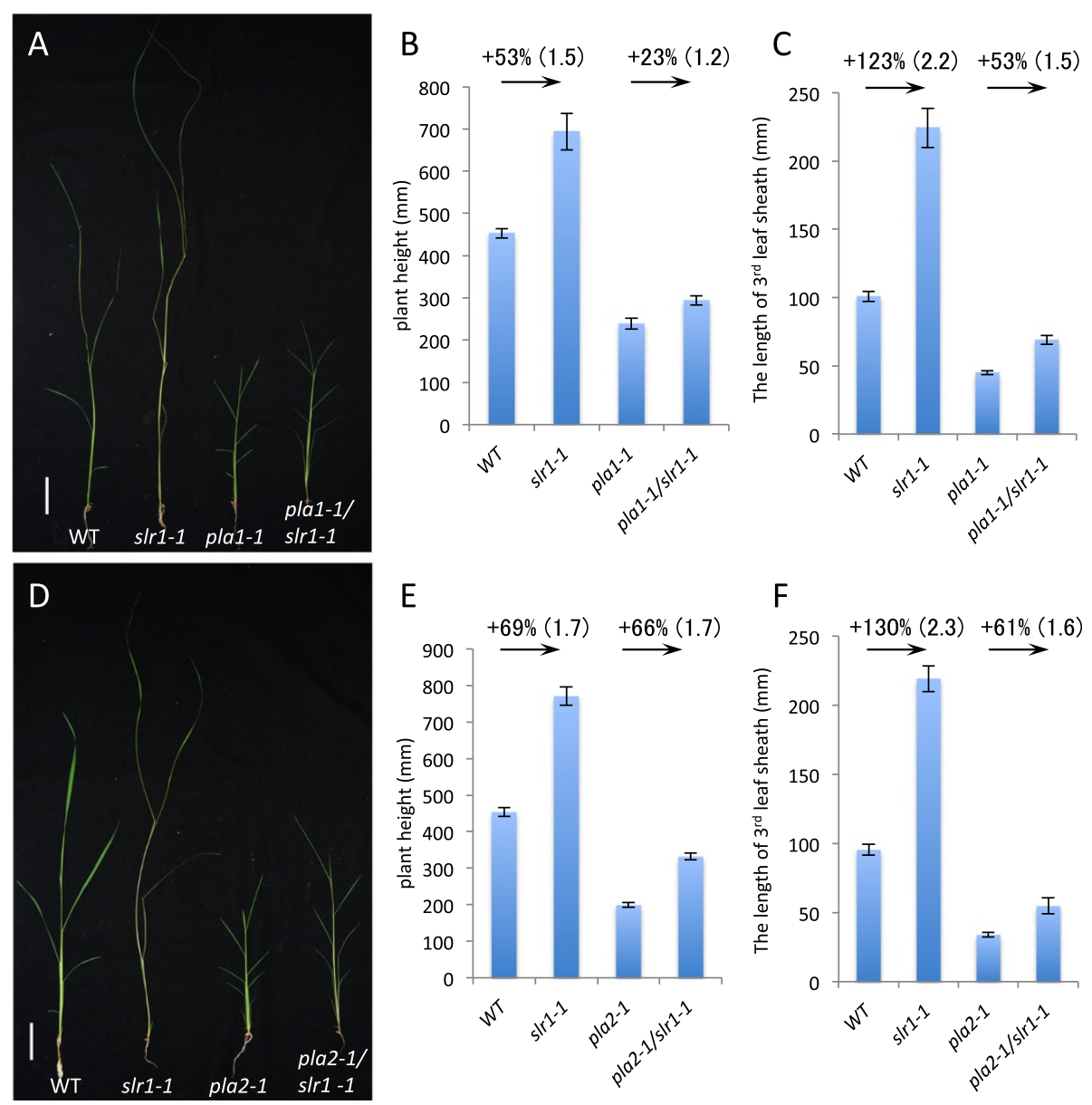

Figure 1 Phenotypes of the pla1/sIr 1 and pla2/sIr 1 double mutants. (A-C) Phenotypes of wild-type, slr 1-1, pla1-1, and pla 1-1/s/r1-1 plants. (D-F) Phenotypes of wild-type, s/r1-1, pla2-1, and pla2-1/s/r1-1 plants. (A, D) Seedlings at 3 weeks after germination (DAG). (B, E) Plant height at 3 weeks after germination. $(\mathbf{C}, \mathbf{F})$ Length of the third leaf sheath. The values represent means \pm SE $(n>5)$. Fold-increase are shown in parenthesis after\% increase. The scale bars indicate $5 \mathrm{~cm}$. 
Table 1 Seedling phenotypes of the pla1-1/s/r1-1 and pla2-1/s/r1-1 double mutants at 3 weeks after germination

\begin{tabular}{|c|c|c|c|c|c|c|c|c|c|c|}
\hline Genotype & $\begin{array}{l}\text { Plant } \\
\text { height }(\mathrm{mm})\end{array}$ & $\begin{array}{l}\text { Leaf } \\
\text { number }\end{array}$ & $\begin{array}{l}\text { 1st Leaf } \\
(\mathrm{mm})\end{array}$ & $\begin{array}{l}\text { 2nd LB } \\
(\mathrm{mm})\end{array}$ & $\begin{array}{l}\text { 2nd LS } \\
(\mathrm{mm})\end{array}$ & $\begin{array}{l}\text { 3rd LB } \\
(\mathrm{mm})\end{array}$ & $\begin{array}{l}\text { 3rd LS } \\
(\mathrm{mm})\end{array}$ & $\begin{array}{l}\text { 4th LB } \\
(\mathrm{mm})\end{array}$ & $\begin{array}{l}\text { 4th LS } \\
(\mathrm{mm})\end{array}$ & $\mathrm{n}$ \\
\hline 1 & $453 \pm 11.3$ & $5.6 \pm 0.16$ & $19.3 \pm 0.6$ & $21.0 \pm 1.4$ & $5.7 \pm 1.7$ & $79.0 \pm 3.9$ & $100.7 \pm 3.5$ & $153.7 \pm 5.5$ & $166.6 \pm 5.4$ & 10 \\
\hline slrt-1 & $693 \pm 43.3$ & $4.8 \pm 0.17$ & $27.8 \pm 2.5$ & $25 \pm 2.6$ & $109.5 \pm 8.5$ & $186.0 \pm 17.5$ & $224.3 \pm 14.4$ & $381.2 \pm 29.8$ & $321.8 \pm 23.1$ & 6 \\
\hline pla1-1 & $38 \pm 13.0$ & $7.8 \pm 0.16$ & $14.9 \pm 0.7$ & $12.6 \pm 1.0$ & $27.6 \pm 1.5$ & $.1 \pm 1.9$ & $45.0 \pm 1.4$ & $35.1 \pm 0.8$ & 1.9 & 8 \\
\hline pla1-1, & $294 \pm 10.8$ & $7.8 \pm 0.31$ & $15.0 \pm 1.2$ & $10.9 \pm 1.5$ & $36.3 \pm 3.7$ & $26.9 \pm 4.1$ & $69.0 \pm 3.2$ & $47.8 \pm 3.3$ & $92.3 \pm 4.4$ & \\
\hline WT & 4 & 5 & 2 & 2 & 8 & 77.3 & 9 & 15 & .7 & \\
\hline slr1-1 & $771 \pm 25.0$ & $5.0 \pm 0.21$ & $30.5 \pm 1.7$ & $25.2 \pm 1.5$ & $109.7 \pm 6.3$ & $162.5 \pm 11.9$ & $219.2 \pm 9.4$ & $396.7 \pm 14.2$ & $344.2 \pm 18.2$ & \\
\hline pla2-1 & $199 \pm 6.7$ & $9.3 \pm 0.15$ & $14.5 \pm 0.9$ & $4.6 \pm 0.5$ & $23.0 \pm 1.6$ & $12.6 \pm 1.0$ & $34.0 \pm 1.7$ & $27.6 \pm 2.2$ & $46.4 \pm 1.5$ & \\
\hline bla2-1/slr1-1 & $332 \pm 8.9$ & $8.4 \pm 0.51$ & $16.8 \pm 1.6$ & $4.8 \pm 0.9$ & $34.4 \pm 4.2$ & $16.8 \pm 1.3$ & $54.8 \pm 5.7$ & $40.0 \pm 4.8$ & $75.4 \pm 5.5$ & \\
\hline
\end{tabular}

LB: leaf blade, LS: leaf sheath. The values indicate the means \pm SE. $n$ indicate the number of seedlings examined in this study.

compared to the corresponding genotypes. These results indicate that PLA1 contributes mainly to cell proliferation in GA-dependent leaf elongation.

\section{Phenotypes of pla2 and s/r1 double mutants}

PLA2 encodes an RNA-binding protein; however, its target RNAs have yet to be elucidated (Kawakatsu et al. 2006). Similar to the pla1/slr 1 double mutants, we examined the phenotype of pla2/slr 1 double mutants. The stature and leaf size of the double mutants were intermediate between those of the pla 2 and $\operatorname{sir} 1$ mutants (Figure 1D-F, Table 1). With regard to plant height, 3week-old $\operatorname{slr} 1$ and pla2/slr 1 seedlings were $69 \%$ and $66 \%$ taller than wild-type and pla 2 seedlings, respectively (Figure 1E). With regard to the length of the third leaf sheath, those of the $\operatorname{slr} 1$ and pla $2 / \operatorname{slr} 1$ plants were $130 \%$ and $61 \%$ longer than in the corresponding genotypes, respectively (Figure 1F). These results suggest that PLA2 is also at least partially involved in GAdependent leaf elongation.

Next, we measured the length of epidermal cells on the adaxial side of the third leaf sheath. The cells of the pla $2 / \operatorname{sir} 1$ double mutant were elongated by $24 \%$ compared to the pla 2 single mutant; whereas those of slr1 were 13\% longer than in wild type (Figure 2B). These results indicate that normal GA-dependent cell elongation occurred in the pla $2 / \operatorname{slr} 1$ double mutants. Accordingly, the suppression of the $\operatorname{slr} 1$ phenotype in the pla2/slr 1 double mutant may have been due to a reduction in cell number.

\section{Expression of genes involved in GA biosynthesis and catabolism in pla/s/r 1 double mutants}

The content of bioactive GA is maintained through feedback regulation (Dai et al. 2007; Olszewski et al. 2002; Yamaguchi 2008). To investigate whether feedback
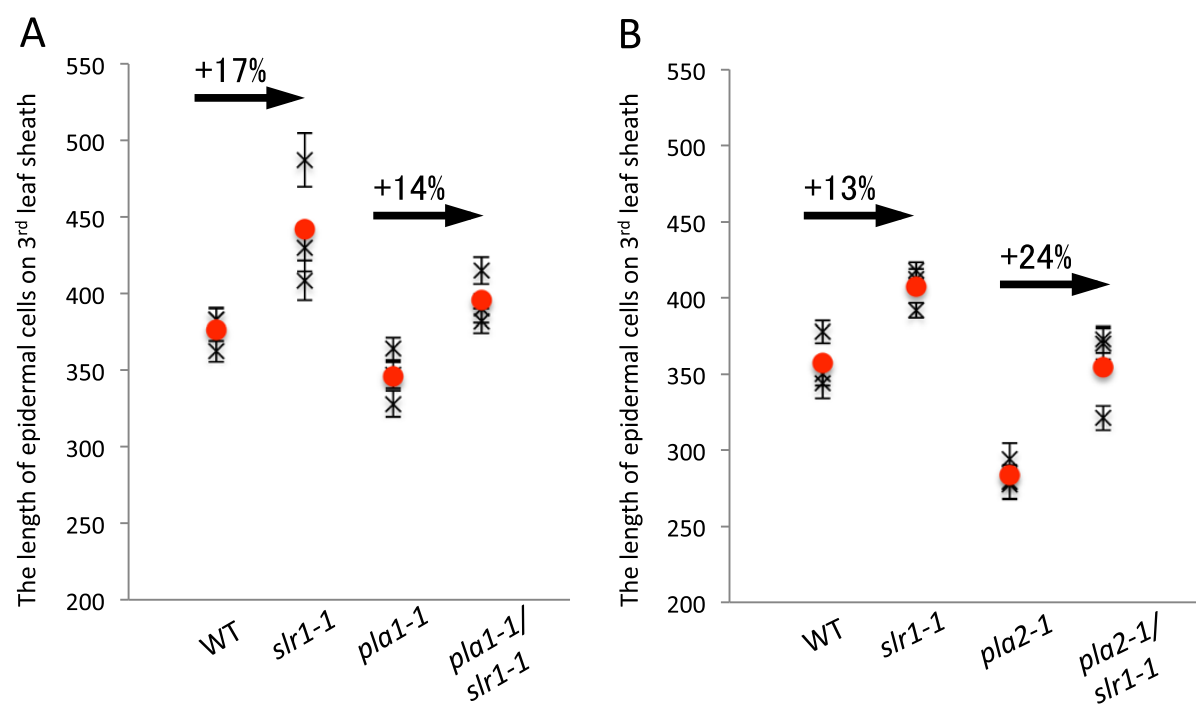

Figure 2 Length of epidermal cells on the adaxial side of the third leaf sheath. (A) Epidermal cell length in wild-type, slr1-1, pla1-1, and pla1-1/s/r1-1 plants. (B) Epidermal cell length in wild-type, s/r1-1, pla2-1, and pla2-1/s/r1-1 plants. Crosses indicate average values of the cells in one sample (average $\pm \mathrm{SE} ; n>100$ ). Closed circles indicate average values of three independent samples. 
regulation for GA homeostasis occurred normally in our pla/slr 1 double mutant plants, we examined the expression levels of two GA biosynthetic genes GA3 oxidase2 (GA3ox2) and GA20 oxidase2 (GA20ox2), and two GA catabolism genes GA2 oxidase1 (GA2ox1) and GA2ox4, in these mutants by real-time PCR (Figure 3, Additional file 1: Table S1). In pla1 and pla2 mutant plants, the expression levels of these GA biosynthetic and GA catabolism genes were comparable to those in wildtype controls. Thus, PLA1 and PLA2 do not affect the expression of genes involved in GA metabolism. The expression of GA3ox2 was slightly decreased in the $\operatorname{sir} 1$ mutant plants, as reported previously (Dai et al. 2007). The expression of GA20ox2 was not decreased in slr1 mutant, indicating that the expression of GA20ox2 may not be under GA feedback regulation in $\operatorname{slr} 1$ mutant. In contrast to GA3ox2 gene, the expression levels of both the GA catabolism genes in $\operatorname{slr} 1$ mutant were increased compared to wild type. Similar to the levels seen in the slr1 mutant, GA3ox2 expression was downregulated and GA2ox1 and GA2ox4 expression was upregulated in the pla1/slr1 and pla2/slr1 double mutant plants. These results indicate that the feedback mechanism and GA response were normal in the pla/slr1 double mutants, at least at the transcriptional level.

GA is involved in various developmental processes, including seed germination, stem elongation, flowering, and pollen maturation (Olszewski et al. 2002; Yamaguchi 2008). Microarray studies have identified several genes involved in the GA pathway (Yazaki et al. 2003; Yang et al. 2004; Jan and Komatsu 2006). However, the genetic regulation downstream of the GA pathway in leaf development is poorly understood. Previous studies suggested that PLA gene products function downstream of GA. In this study,
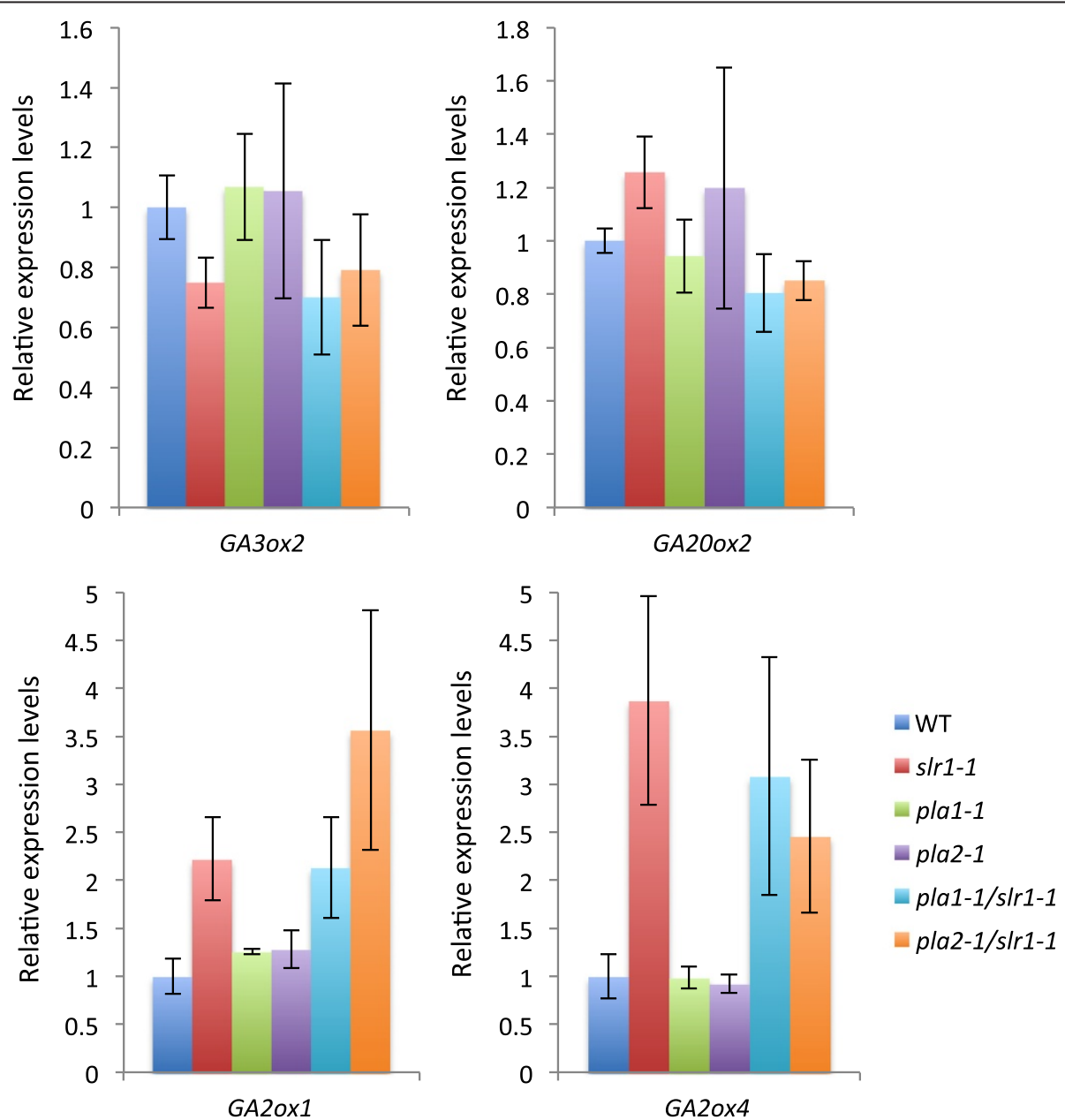

Figure 3 Relative expression levels of GA biosynthetic (GA3ox2, GA20ox2) and GA catabolism (GA2ox1, GA2ox4) genes in wild-type, slr1-1, pla1-1, pla2-1, pla1-1/slr1-1, and pla2-1/slr1-1 plants. The expression levels in the mutants are represented relative to that in wild type (assigned a value of 1). The values indicate the means of three biological samples \pm SE. Actin1 was used as an internal control. GA30x2, GA20ox2, GA20X4 and Actin 1 were quantified using TaqMan probes. GA2OX1 was quantified by SYBR green. The primers and probes for each gene are listed in Additional file 1: Table S1. 
we demonstrated genetic interactions between $P L A$ genes and $S L R 1$, a central regulator of GA signaling, supporting our previous results.

Our analysis suggests that the intermediate phenotypes of the pla/slr1 double mutants were probably due to a reduction in cell number. There are two explanations for why the absence of $P L A$ functions partly suppressed the slr1 phenotype. First, PLA genes are involved in cellular proliferation in the GA-dependent pathway. Recent studies have indicated that GA promotes not only cell expansion but also cellular proliferation through the regulation of cell cycle inhibitor genes (Achard et al. 2009). In addition, GA controls the transition from cell proliferation to expansion in maize leaves (Nelissen et al. 2012). Thus, GA can influence cell number during leaf development, and it is possible that PLA functions are required for cell proliferation rather than cell elongation downstream of the GA pathway. Second, defects in PLA genes affect the duration and/or timing of cellular proliferation, resulting in a decrease in the total cell number in the leaves of pla/slr1 mutants. Previous studies suggested that PLA1 and PLA2 genes regulate the rate of leaf maturation (Kawakatsu et al. 2006) and that the small leaves in pla mutants were due to precocious leaf maturation. Thus, it is possible that the duration of cell proliferation in developing leaves is insufficient in pla/slr1 double mutants, resulting in suppression of the $\operatorname{slr} 1$ phenotype.

Our results indicate that PLA genes partly regulate leaf size by affecting cell proliferation via the GA-dependent pathway. However, it remains unclear how PLA genes regulate cellular proliferation in the GA signaling pathway. Further study is required to clarify the molecular mechanisms underlying the regulatory roles of $P L A$ gene expression in GA-dependent leaf development.

\section{Additional file}

Additional file 1: Table S1. List of primers used in real-time PCR assays.

\section{Abbreviations}

GA: Gibberellin; PLA: PLASTOCHRON; SLR1: SLENDER RICE1.

\section{Competing interests}

The authors declare that they have no competing interests.

\section{Authors' contributions}

MM performed the experiments and drafted the manuscripts. JI conceived the study and drafted manuscripts. Both authors read and approved the final manuscript.

\section{Acknowledgements}

We would like to thank Dr. H. Kitano (Nagoya University) for kind gift of s/r 1-1 seeds. We also thank R. Soga and K. Ichikawa (The Institute for Sustainable Agro-ecosystem Services, University of Tokyo) for their assistance in cultivating rice plants at the Experimental Farm of the University of Tokyo. This work was partially funded by JSPS KAKENHI 24380005 (J.I.) and Grant-in-Aid for JSPS Fellows 25-8135 (M.M.)
Received: 17 June 2014 Accepted: 11 September 2014

Published online: 18 September 2014

\section{References}

Anastasiou E, Kenz S, Gerstung M, MacLean D, Timmer J, Fleck C, Lenhard M (2007) Control of plant organ size by KLUH/CYP78A5-dependent intercellular signaling. Dev Cell 13:843-856

Achard P, Gusti A, Cheminant S, Alioua M, Dhondt S, Coppens F, Beemster GT, Genschik P (2009) Gibberellin signaling controls cell proliferation rate in Arabidopsis. Curr Biol 19:1188-1193

Adamski NM, Anastasiou E, Eriksson S, O'Neill CM, Lenhard M (2009) Local maternal control of seed size by KLUH/CYP78A5-dependent growth signaling. Proc Natl Acad Sci U S A 106:20115-20120

Chakrabarti M, Zhang N, Sauvage C, Muños S, Blanca J, Cañizares J, Diez MJ, Schneider R, Mazourek M, McClead J, Causse M, van der Knaap E (2013) A cytochrome $\mathrm{P} 450$ regulates a domestication trait in cultivated tomato. Proc Natl Acad Sci U S A 110:17125-17130

Dai M, Zhao Y, Ma Q, Hu Y, Hedden P, Zhang Q, Zhou DX (2007) The rice YABBY1 gene is involved in the feedback regulation of gibberellin metabolism. Plant Physiol 144:121-133

Eriksson S, Stransfeld L, Adamski NM, Breuninger H, Lenhard M (2010) KLUH/ CYP78A5-dependent growth signaling coordinates floral organ growth in Arabidopsis. Curr Biol 20:527-532

Fang W, Wang Z, Cui R, Li J, Li Y (2012) Maternal control of seed size by EOD3/ CYP78A6 in Arabidopsis thaliana. Plant J 70:929-939

Ikeda A, Ueguchi-Tanaka M, Sonoda Y, Kitano H, Koshioka M, Futsuhara Y, Matsuoka M, Yamaguchi J (2001) Slender rice, a constitutive gibberellin response mutant, is caused by a null mutation of the SLR1 gene, an ortholog of the height-regulating gene GAI/RGA/RHT/D8. Plant Cell 13:999-1010

Jan A, Komatsu S (2006) Functional characterization of gibberellin-regulated genes in rice using microarray system. Genomics Proteomics Bioinformatics 4:137-144

Kawakatsu T, Itoh J, Miyoshi K, Kurata N, Alvarez N, Veit B, Nagato Y (2006) PLASTOCHRON2 regulates leaf initiation and maturation in rice. Plant Cell 18:612-625

Mimura M, Nagato Y, Itoh J (2012) Rice PLASTOCHRON genes regulate leaf maturation downstream of the gibberellin signal transduction pathway. Planta 235:1081-1089

Miyoshi K, Ahn BO, Kawakatsu T, Ito Y, Itoh J, Nagato Y, Kurata N (2004) PLASTOCHRON1, a timekeeper of leaf initiation in rice, encodes cytochrome P450. Proc Natl Acad Sci U S A 101:875-880

Nelissen H, Rymen B, Jikumaru Y, Demuynck K, Van Lijsebettens M, Kamiya Y, Inzé $D$, Beemster GT (2012) A local maximum in gibberellin levels regulates maize leaf growth by spatial control of cell division. Curr Biol 22:1183-1187

Olszewski N, Sun TP, Gubler F (2002) Gibberellin signaling: biosynthesis, catabolism, and response pathways. Plant Cell 14:S61-S80

Sotelo-Silveira M, Cucinotta M, Chauvin AL, Chávez Montes RA, Colombo L, Marsch-Martínez N, de Folter S (2013) Cytochrome P450 CYP78A9 is involved in Arabidopsis reproductive development. Plant Physiol 162:779-799

Yamaguchi S (2008) Gibberellin metabolism and its regulation. Annu Rev Plant Biol 59:225-251

Yang GX, Jan A, Shen SH, Yazaki J, Ishikawa M, Shimatani Z, Kishimoto N, Kikuchi S, Matsumoto H, Komatsu S (2004) Microarray analysis of brassinosteroidsand gibberellin-regulated gene expression in rice seedlings. Mol Genet Genomics 271:468-378

Yazaki J, Kishimoto N, Nagata Y, Ishikawa M, Fujii F, Hashimoto A, Shimbo K, Shimatani Z, Kojima K, Suzuki K, Yamamoto M, Honda S, Endo A, Yoshida Y, Sato Y, Takeuchi K, Toyoshima K, Miyamoto C, Wu J, Sasaki T, Sakata K, Yamamoto K, Iba K, Oda T, Otomo Y, Murakami K, Matsubara K, Kawai J, Carninci P, Hayashizaki Y, Kikuchi S (2003) Genomics approach to abscisic acid- and gibberellin-responsive genes in rice. DNA Res 10:249-261

doi:10.1186/s12284-014-0025-2

Cite this article as: Mimura and Itoh: Genetic interaction between rice PLASTOCHRON genes and the gibberellin pathway in leaf development. Rice 2014 7:25 